ARTICLE

\title{
A Method for Measuring LC Resonance Frequency by Impulse Response
}

\section{Mingwei $\mathrm{Xu}^{*}$ Jiuxin Gong Yuechang Shi}

School of Information Engineering, North China University of Science and Technology, Tangshan, Hebei, 063210, China

\begin{tabular}{l}
\hline ARTICLE INFO \\
\hline Article history \\
Received: 17 January 2020 \\
Revised: 24 January 2020 \\
Accepted: 9 April 2020 \\
Published Online: 16 April 2020 \\
\hline
\end{tabular}

\section{Keywords:}

LC circuit

Resonant frequency

Digital Storage Oscilloscope

Impulse signal

\section{Measurement Principle}

$\mathrm{T}$ This system provides an instantaneous impulse current to the excitation coil to generate a large instantaneous magnetic field. According to the law of electromagnetic induction, the LC circuit under test placed inside the excitation coil will induce an initial impulse current. This initial energy Under-damped oscillation will be generated in the $\mathrm{LC}$ circuit. The resonance frequency of this LC circuit can be analyzed by grabbing a piece of oscillation waveform through a digital storage oscilloscope. It is easy to use and has low dependence on high-end instruments. The disadvantage is that the accuracy is limited and it basically depends on the oscilloscope Frequency resolution.

\section{Test System Composition}

The test system shown in figure 1 is mainly composed of an excitation coil, an impulse current generator, an LC circuit to be tested and a digital storage oscilloscope.
ABSTRACT

LC circuit resonance frequency measurement often requires the use of professional analysis instruments such as LCR meters, vector network analyzers, but currently such instruments on the market are expensive, and it is difficult for non-professional institute personnel to access. Here comes unnecessary trouble. In view of this situation, a test method for measuring the resonance frequency using only a digital storage oscilloscope is proposed. Using the impulse signal to obtain the system response, the response waveform period can be observed through the oscilloscope.

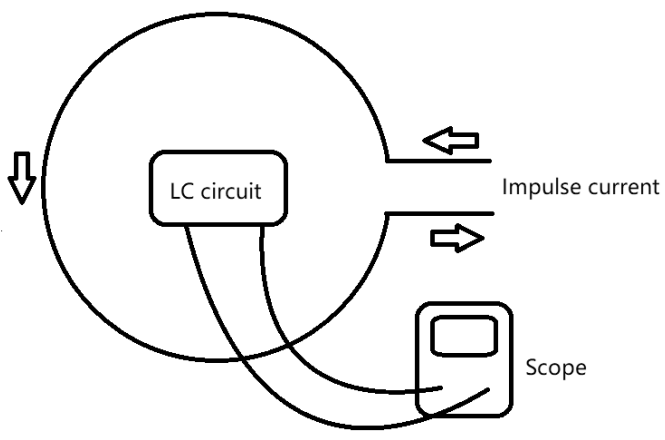

Figure 1. Structure of the test system

\subsection{Excitation Coil}

The material selection of the excitation coil is based on the internal resistance as a selection criterion. It is better to choose a conductor material that is easy to obtain and has a small internal resistance to allow a large current to pass. In this experiment, a $1.2 \mathrm{~mm}$ diameter copper enam-

*Corresponding Author:

Mingwei Xu,

School of Information Engineering, North China University of Science and Technology, Tangshan, Hebei, 063210, China;

E-mail:xumingweiaa@qq.com. 
eled wire was used to surround the excitation coil.

Since the measurement of the resonance frequency of the LC circuit to be measured is obtained by the voltage waveform generated by its oscillation, and most of the ADCs on the market use 8-bit AD in the internal ADC, the vertical resolution is poor, so the $\mathrm{LC}$ circuit the larger the voltage peak generated in the better. Since this system uses the principle of electromagnetic induction to generate an oscillating current in the LC circuit, the larger the voltage generated in the circuit, the greater the magnetic induction intensity generated in the excitation coil. In this experiment, one turn of enameled wire was used to energize the coil.

\subsection{Inrush Current Generator}

After the number of coil turns is fixed, according to Biot-Savart's law, the magnetic induction intensity generated by the coil is related to the magnitude of the excitation current. The formula is as follows:

$$
\vec{B}=\int_{L} \frac{\mu_{0} I}{4 \pi} \frac{d l \times \overrightarrow{e_{r}}}{r^{2}}
$$

At the same time, in order to ensure that the excitation current input to the LC system is close to the impulse signal, it is necessary to provide a high-current, short-time narrow pulse signal to approximate the impulse signal. In this experiment, a $10000 \mathrm{uF}$ electrolytic capacitor is used to provide instantaneous high current, and a set of relays and MCUs are used to precisely control the charging and discharging of the electrolytic capacitor. The control circuit is shown in figure 2. The relay JK1 controls the charging of the electrolytic capacitor, and the relay JK2 controls the discharge of the electrolytic capacitor. It is necessary to ensure that the two relays cannot be turned on at the same time during charging and discharging, and add a dead time when the state is switched. Failure to do so will cause a short circuit and burn out the relay and excitation coil.

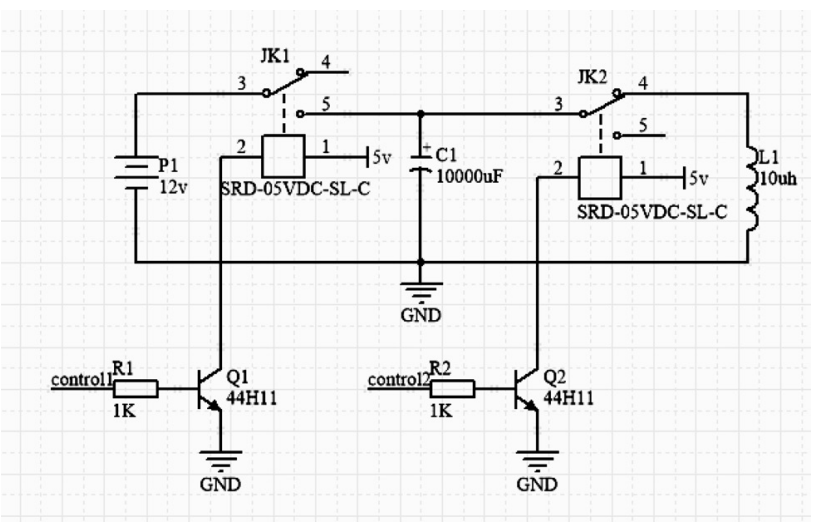

Figure 2. Inrush current control circuit

\subsection{LC Circuit under Test}

Because this system uses the oscilloscope to capture the voltage waveform of the LC circuit, it is necessary to connect the capacitor and the inductor in parallel to form a series resonance. In this way, you can connect an ordinary oscilloscope probe to both ends of the capacitor or inductor to capture the waveform, as shown in the figure. As shown in figure 3.

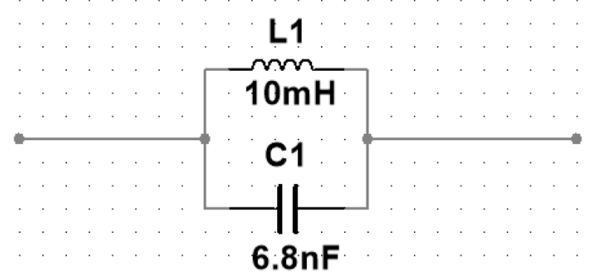

Figure 3. LC circuit

In this experiment, a $10 \mathrm{mH}$ I-shaped inductor as shown in figure 4 and a $6.8 \mathrm{nF}$ high-frequency capacitor as shown in figure 5 are used to form an LC circuit.

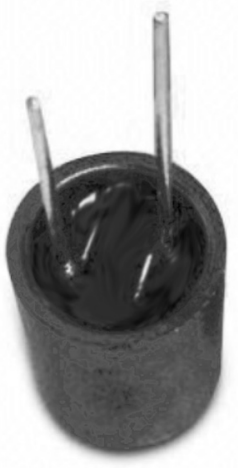

Figure 4. I-shaped inductor

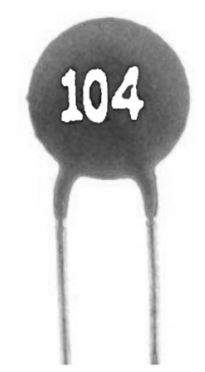

Figure 5. High-frequency capacitor

\section{Measurement Steps}

\subsection{Step 1}

Connect the excitation system and check it correctly. Place 
the LC circuit under test in the center of the excitation coil. Because the direction of the magnetic induction lines generated in the excitation coil is perpendicular to the horizontal plane where the coil is located, in order to maximize the magnetic field energy received by the LC circuit, it is necessary to make these magnetic induction lines pass through the largest number of I-shaped inductors. When the inductor is placed perpendicular to the plane where the excitation coil is located, the magnetic induction line can pass horizontally through the coil inside the I-shaped inductor to maximize the magnetic field energy obtained. If the inductor is mistakenly prevented from being parallel to the plane where the excitation coil is located, the magnetic field energy is basically not obtained, and the oscilloscope will not capture an obvious voltage waveform.

\subsection{Step 2}

Set the trigger mode of the digital storage oscilloscope to single trigger mode, turn on the cursor mode, and set the cursor to the tracking mode. After the oscilloscope has set the single trigger mode, it needs an initial trigger level to trigger capture. After the trigger, the oscilloscope will capture the waveform for a period of time. This initial trigger level is replaced by the voltage signal in the LC circuit.

\subsection{Step 3}

Firstly, the MCU controls the capacitor to be charged, and then controls the capacitor to instantaneously discharge the excitation coil to generate an instantaneous impulse current in the coil. At the same time, the oscilloscope will capture a period of oscillation waveform as shown in figure 6. When the oscilloscope is set after the cursor-tracking mode, for the under-damped oscillation waveform obtained after a single trigger, the time difference between two adjacent peaks or troughs is automatically measured, which is the oscillation period, and the resonant frequency of the LC circuit can be obtained by conversion.

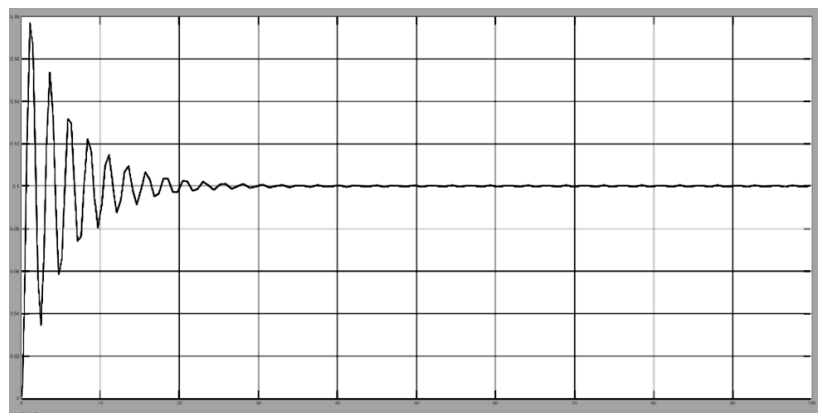

Figure 6. Underdamped Oscillation Waveform

\section{Conclusion}

Aiming at the method of measuring LC resonance frequency of low-end instruments, this paper proposes a simpler and lower cost measurement method. By setting up a simple test circuit and a low-end digital storage oscilloscope, the LC resonance frequency can be measured more conveniently, but the measurement accuracy often depends on the frequency resolution of the oscilloscope. This also becomes one of the reasons for measurement errors. The result is basically the same as the test result using the LCR tester, and it can be used under limited economic conditions.

\section{References}

[1] Resonance characteristics of RLC measured by digital oscilloscope [J]. Li Chaorui. Physical Experiment. 2018 (04)

[2] Li Chaorui.Intuitive measurement method of LC resonance parameters [J] .Physics Experiment, 2019,39 (04): 32-35. 